University of Wollongong

Research Online

Faculty of Social Sciences - Papers (Archive) Faculty of Arts, Social Sciences \& Humanities

2013

Factor analysis and validity of a short six-item version of the Desires for Alcohol Questionnaire

C Mo

University of Wollongong

Frank P. Deane

University of Wollongong, fdeane@uow.edu.au

Geoffrey C. Lyons

University of Wollongong, glyons@uow.edu.au

Peter Kelly

University of Wollongong, pkelly@uow.edu.au

Follow this and additional works at: https://ro.uow.edu.au/sspapers

Part of the Education Commons, and the Social and Behavioral Sciences Commons

Research Online is the open access institutional repository for the University of Wollongong. For further information contact the UOW Library: research-pubs@uow.edu.au 


\title{
Factor analysis and validity of a short six-item version of the Desires for Alcohol Questionnaire
}

\begin{abstract}
Reductions in cravings have been associated with improved recovery from alcohol and other drug use problems. Self-report assessments of cravings provide a way of monitoring progress over the course of treatment particularly in residential settings. There is a need to develop brief craving measures suitable for repeat administration. The aim of the study was to assess the reliability and validity of a six-item version of the Desires for Alcohol Questionnaire (DAQ-6). In study 1 exploratory factor analysis involving 710 participants attending residential treatment revealed two factors: 'expectancy of negative reinforcement' and 'strong desires and intentions'. In study 2 confirmatory factor analysis replicated this factor structure in an independent sample of 116 participants. Both studies provided evidence for convergent and discriminant validity of the DAQ- 6 when compared to other measures. The DAQ- 6 shows promise as a brief self-report measure of cravings but the utility of the separate subscales in treatment contexts requires further research.
\end{abstract}

\section{Keywords}

version, item, questionnaire, desires, six, factor, short, validity, alcohol, analysis

\section{Disciplines}

Education | Social and Behavioral Sciences

\section{Publication Details}

Mo, C., Deane, F. P., Lyons, G. C. \& Kelly, P. (2013). Factor analysis and validity of a short six-item version of the Desires for Alcohol Questionnaire. Journal of Substance Abuse Treatment, 44 (5), 557-564. 


\section{Running head: FACTOR ANALYSIS AND VALIDITY OF A SHORT 6-ITEM VERSION}

Factor Analysis and Validity of a Short 6-Item Version of the Desires for Alcohol Questionnaire

Carol Mo, Frank P. Deane, Geoffrey, C. B. Lyons \& Peter J. Kelly

University of Wollongong, Australia

Author for correspondence: Frank Deane, School of Psychology and Illawarra Institute for Mental Health, Building 22, University of Wollongong, Wollongong, NSW 2522, Australia.

Telephone: 02 4221 4523, Fax: 02 4221 5585, Email: fdeane@uow.edu.au

Section of journal: Full-length article

Word Count: 4772 


\begin{abstract}
Reductions in cravings have been associated with improved recovery from alcohol and other drug use problems. Self report assessments of cravings provide a way of monitoring progress over the course of treatment particularly in residential settings. There is a need to develop brief craving measures suitable for repeat administration. The aim of the study was to assess the reliability and validity of a 6-item version of the Desires for Alcohol Questionnaire (DAQ-6). In Study 1 exploratory factor analysis involving 710 participants attending residential treatment revealed two factors: 'Expectancy of Negative Reinforcement' and 'Strong Desires and Intentions'. In Study 2 confirmatory factor analysis replicated this factor structure in an independent sample of 116 participants. Both studies provided evidence for convergent and discriminant validity of the DAQ-6 when compared to other measures. The DAQ-6 shows promise as a brief self report measure of cravings but the utility of the separate subscales in treatment contexts requires further research.
\end{abstract}

Keywords: craving; measure; psychometrics; factor analysis 


\section{Introduction}

The definition and measurement of alcohol and drug 'craving' has been the topic of debate for many decades in research and treatment settings (Drobes \& Thomas, 1998; World Health Organisation, 1955). Although craving has been defined in a number of ways, it is commonly understood as a persistent urge or desire to use a substance (American Psychiatric Association, 2000; Kozlowski \& Wilkinson, 1987). Theories of addiction have elaborated on this definition by positing that craving is central to substance dependence and relapse, and is generally argued to arise from the positive-reinforcing outcomes of a substance, the negativereinforcing effects related to substance withdrawal, or a combination of both (e.g. Baker, Piper, Fiore, McCarthy, \& Majeskie, 2004; Marlatt, 1985; Marlatt \& Gordon, 1985; Niaura, 2000; Niaura et al., 1988).

In addition to its theoretical importance, the role of craving provides many practical benefits to researchers and clinicians (Drummond Litten, Lowman \& Hunt, 2000; Rosenberg, 2009). Some of these benefits include: 1) the ability to make predictions regarding the readiness for treatment discharge and the likelihood of relapse following discharge, 2) recommendations regarding appropriate treatment based on craving profiles, and 3) the development of new treatments aimed at preventing relapse by reducing craving itself, or helping patients to cope more effectively with craving. Given these potential benefits, reliable and valid measures of craving are important in both research and treatment contexts. This is particularly relevant in residential settings because abstinence is often a requirement for patients. Consequently, measures typically used to track progress in outpatient settings, such as quantity or frequency of substance use, are not suitable. 
To date, there is still no consensus on an optimal measure of craving (Rosenberg, 2009; Sayette et al., 2000). Traditional measures of craving tended to rely on single-item visual analogue scales to measure the severity of craving (McCormick, Home, \& Sheather, 1988). While single-item scales are easy to administer and suitable for repeated use, they are problematic because of questionable reliability and furthermore, they dismiss the multidimensional nature of craving (Anton \& Drobes, 1998; McCormick et al., 1988). Interestingly, over the last two decades, researchers have addressed these issues by developing lengthy multi-item questionnaires, but have since reduced the number of items in order to retain the advantages of shorter questionnaires (see Rosenberg, 2009). Examples of questionnaires that have followed this trend include: the Questionnaire of Smoking Urges (QSU; Tiffany \& Drobes, 1991), the Obsessive Compulsive Drinking Scale (OCDS; Anton, Moak, \& Latham, 1995), and the Desires for Alcohol Questionnaire (DAQ; Clark, 1994) (see Rosenberg, 2009 for a list of multi-item self-report craving questionnaires).

The DAQ, which is the focus of the present study, is one questionnaire that has undergone multiple revisions to determine the optimal number of items and dimensions that capture craving. The original DAQ (Clark, 1994) included 36 items that aimed to assess craving for alcohol. Factor analyses of 302 participants revealed four factors that accounted for $65 \%$ of the overall variance. Factors included: "alleviation of negative affect", "strong desires and intentions", "mild intentions and positive reinforcement" and "controllability of alcohol consumption once drinking is initiated”. The 36-item was subsequently reduced to a 14-item version by selecting items that loaded highly on each dimension (see Kramer et al., 2010; Love et al., 1998). 
Love et al. (1998) administered the 14-item DAQ to 131 participants who were attending treatment for alcohol use. Results revealed four factors that explained $81.5 \%$ of the overall variance: “negative reinforcement”, “control over drinking”, “strong desires and intentions to drink", and “mild desires and intentions to drink”. However, items relating to mild desires loaded alongside those relating to strong desires. This suggests that participants with more severe alcohol problems are less able to distinguish between the intensity of their intentions to drink. Thus, “mild desires” may be a less appropriate factor in this population. Similarly, Kramer et al. (2010) also administered this version of the DAQ to 1500 participants with an alcohol-use disorder (AUD) and 1460 without an AUD. In both subsamples, results revealed a three-factor solution that accounted for over $70 \%$ of the overall variance. Items reflected "strong intentions and desire to use”, “negative reinforcement”, and "positive reinforcement and ability to control drinking”. However, they did not reflect mild desires and drinking control was not clearly distinguished from positive reinforcement.

The DAQ has also been used for specific individual substances other than alcohol and shown to have similar craving factor structures. One study developed the Desire for Drug Questionnaire by modifying the 14-item DAQ for heroin-dependent patients (Franken, Hendriks, \& van den Brink, 2002). Another study used the original DAQ to develop the Desire of Speed Questionnaire to measure craving for amphetamines and found four factors that were almost identical to the original DAQ factors (James, Davies, \& Willner, 2004). However, major limitations of this study were the use of a convenience sample which limits generalisability and, presence of a large number of items that did not fit any of the factors due to high cross loadings on multiple factors. 
Closer inspection of the studies reviewed indicates that while the original DAQ proposed four factors that explain craving, subsequent studies have reported two consistent dimensions. These factors are related to strong desires and intentions to use and the reinforcing effects of using the substance. Items related to "mild desires" to use tend to load alongside "strong desires and intentions”. Several studies have also found a two item "control” factor (Franken et al., 2002; James et al., 2004; Love et al., 1998), but one study found that items from this factor loaded on the "positive reinforcement” factor (Kramer et al., 2010). Given that high Cronbach’s alpha has been reported even for the 14-item version (range 0.95 to 0.97) (Love et al., 1998), this indicates some degree of item redundancy. Even relatively short questionnaires can overwhelm participants and increase response errors, particularly if they are administered routinely as part of a larger battery of questionnaires (Marteau \& Bekker, 1992; Myers et al., 2003; Taylor \& Deane, 2002). Thus, an even briefer version of the DAQ that includes cravings for both alcohol and other drugs would better reflect the mixed background of participants in residential treatment settings and reduce completion times and burden.

\subsection{The Present Study}

Two studies were conducted to assess the reliability and validity of a brief 6-item version of the DAQ. In Study 1 the exploratory factor analysis was conducted on the 6-item DAQ in a large sample of individuals attending residential drug and alcohol treatment. Convergent validity was assessed by examining the relationship between this version of the DAQ and other measures related to craving that are typically used in SUD contexts. It is expected that this craving measure will correlate positively with measures of symptom distress and negatively with measures of recovery. In Study 2, confirmatory factor analysis (CFA) was conducted on an 
independent sample of participants from similar settings in order to determine whether the same factor structure arises. In addition, convergent and divergent validity was assessed.

Study 1

2. Material and Methods

\subsection{Participants}

Participants were recruited from eight Salvation Army alcohol and drug rehabilitation centres located along the east coast of Australia. The program utilises a 12-Step, faith-based and abstinence-oriented approach to provide up to 10 months of residential treatment to individuals with substance use disorder (SUD). Participants in this study were selected from the cohort of clients who arrived at any of the centres for treatment during the period of June 2008 to July 2010 inclusive. The total number of new admissions and potential participant pool over this time was 1271. Additionally, participants were eligible if they: (i) provided informed consent and (ii) completed the Desire for Alcohol Questionnaire within one week of completing the Addiction Severity Index at treatment entry. Given that craving decreases with abstinence, it was important to ensure that craving was measured as close to treatment entry as possible (e.g. Doherty, Kinnunen, Militello, \& Garvey, 1995; McGregor et al., 2005). A total of 712 participants met these criteria, resulting in a 56\% participation rate. This sample comprised 579 males (81\%) and 133 females (19\%). This is consistent with the proportion of beds allocated to males and females across The Salvation Army treatment sites. The average age of participants was 36.75 ( $S D=10.76)$. The primary substance of use was alcohol (59.7\%) and the average length of problem was 18.38 years $(S D=10.99)$. This was followed by amphetamines $(14.4 \%)$, cannabis (12.6\%), and heroin (5.8\%). 


\subsection{Development of DAQ-6}

The 36-item and 14-item DAQ are the two most common versions used to measure craving in research. We did not include all items in the initial design because the research context was such that we needed a brief version of a craving measure to reduce the burden on participants since there were multiple other measures being simultaneously administered as part of routine outcomes assessment in The Salvation Army treatment centres. Thus, the current study is based on secondary analysis of routine outcome data. As discussed, items from the longer versions of the DAQ consistently fall into the dimensions of "strong desire and intention to use" and “negative reinforcement”. Based on such findings, 3 items that loaded highly on each dimension, which were also relevant to residential treatment settings, were chosen. Some studies have included a third "control” factor, but we did not include this factor in part because we based our item selection primarily on the factor analysis of Kramer et al. (2010) who found these control items cross-loaded on another factor. Particular reference was paid to the factor loadings generated from the study by Kramer et al., (2010), where the same items had the highest factor loadings on the two aforementioned factors. These items were worded identically to the original DAQ with the only difference being the replacement of alcohol to include drugs (e.g. drink/ use drugs). Participants were prompted to select their drug of preference and then to rate how strongly they agreed or disagreed with each statement on a 7-point Likert Scale ranging from “Strongly disagree” to "Strongly agree”. Examples of items used in this study include: “I want to drink/ use drugs so much I could taste it" and "I would feel as if all the bad things in my life disappeared if I drank/ used drugs now”.

\subsection{Measures}


The Drug Taking Confidence Questionnaire (DTCQ) (Sklar, Annis \& Turner, 1999) is an 8-item self-report questionnaire that measures a person's self-efficacy in resisting the urge to drink alcohol or take drugs in specific high risk relapse situations. Participants are asked to rate their confidence on a scale from 0 to 100\%, and then scores are averaged to form an overall confidence percentage. Examples of items included asking the participant their confidence to resist the urge to use their primary drug if they felt "angry", had "trouble sleeping” and wanted to “celebrate”. The construct validity of the DTCQ has been supported by positive correlations with measures of confidence and motivation to quit drinking or using drugs, and negative correlations with a measure of difficulty quitting alcohol and drugs (Sklar \& Turner, 1999). Cronbach’s alpha for the DTCQ has been reported as .89 (Sklar \& Turner, 1999), and in the current study it was 0.90.

The Addiction Severity Index, fifth edition (ASI-5) (McLellan et al., 1992) is a semistructured interview used to assess the severity of an individual's health status in 7 domains: Medical Status, Employment/ Support Status, Alcohol and Drug Use, Legal Status, Family/ Social Relationships and Psychiatric Status. Questions are asked about the extent of these problems in the individual's lifetime and the past 30 days. The ASI produces a composite score ranging from 0 (no problem) to 1 (severe problem) for each domain. Each composite score indicates the severity of that domain for the past 30 days. The complete ASI was administered, however for the purpose of this study only Alcohol Use and Drug User domains were used.

The Depression Anxiety Stress Scale 21 (DASS-21) (Lovibond \& Lovibond, 1995) is a 21 item self-report questionnaire that consists of three scales that measure negative emotional states of depression, anxiety and stress. Items are scored on a 4-point Likert Scale from 'Did not apply to me at all' (0) to 'Applied to me very much or most of the time' (3) (e.g. 'I find it hard to 
wind down'). Cronbach's alpha for the total score has been reported as .93 (Henry \& Crawford, 2005), and in the current study it was 0.96 .

The Mental Health Continuum-Short Form (MHC-SF) (Lamers, Westerhof, Bohlmeijer, ten Klooster, \& Keves, 2011) measures participants' feelings of psychological, emotional and social well-being over the past month. Participants rate the frequency of 14 feeling states on a 6point Likert scale range from 'Never’ (9) to ‘Every day’ (5). Example of items include ‘Happy’, 'Interested in life', and 'That people are basically good'. Research has confirmed the reliability and validity of the MHC-SF as a measure of positive mental health and wellbeing, with Cronbach’s alpha reported as 89 (Lamers et al., 2011). The Cronbach alpha in the current study was 0.94 .

The Recovery Assessment Scale (RAS-24) (Corrigan, Salzer, Ralph, Sangster \& Keck, 2004) is a 24 item scale which asks participants to indicate the extent to which they agree with statements about themselves and their lives. It assess: Personal Confidence and Hope, Willingness to ask for Help, Goal and Success Orientation, Reliance on Others and Symptom Coping and No Domination of Symptoms. Items are scored on a 5-point Likert scale ranging from 'Strongly disagree' (1) to 'Strongly agree' (5), with items such as 'I have a desire to succeed'. Items are summed to produce a total score, with higher scores indicating greater recovery. The Cronbach alpha for the whole scale in the current study was 0.92.

The Life Engagement Test (LET) (Scheier et al., 2006) is a 6-item scale that measures purpose in life. Items are scored on a 5-point Likert scale ranging from 'Strongly disagree' (1) to 'Strongly agree' (5), with items such as 'There is not enough purpose in my life' (item 1). The reliability of this scale tested across eight samples ranges from .72 to .87 (Scheier et al., 2006). The Cronbach alpha for this study was 0.78 . 


\subsection{Procedure}

The intake assessment, which included all the aforementioned measures, was administered during the first admission session conducted at the residential treatment centres. All assessments were conducted by The Salvation Army caseworkers and then all data collected was entered into their electronic file management program. The study protocols were reviewed and approved by the University of Wollongong Human Ethics Committee.

\subsection{Statistical analysis}

The data collected from the DAQ-6 were analysed using exploratory factor analyses. Only participants that completed questionnaires within seven days of treatment entry were included in the analysis. There were small amounts of missing data and this resulted in 710 cases being retained for the Principal components analysis (PCA). Obtained components of the PCA were subjected to oblique (promax) rotation as previous studies reported intercorrelations among components of craving. The number of factors retained was determined by inspection of scree plots and eigenvalues, as well as interpretability of each component. Additionally, only items with loadings higher or equal to 0.4 were considered part of that component. The reliability of resulting factor scales were checked using Cronbach’s alpha. The data were checked for sampling adequacy and sphericity using the Kaiser-Meyer-Olkin (KMO) and Bartlett tests. The KMO measure in this study was 0.83 , which is well above the acceptable limit of .5 . Additionally, Barlett's test of sphericity indicated that correlations between items were sufficiently large and therefore factor analysis is appropriate $\left(\chi^{2}(15)=2745.56, p<.001\right)$.

Correlations were conducted between the various measures of psychological functioning and drug and alcohol specific variables in order to assess convergent and divergent validity of the DAQ-6. Only a subsample of participants completed the additional measures and analyses for 
some variable were restricted to only those who endorsed the specific problem (e.g., ASI Drug composite) so sample sizes ranged between 232 and 382 for these pairwise comparisons.

\section{Results}

\subsection{Factor analysis}

Factor analysis revealed two factors with eigenvalues of 3.87 and 1.03. Three items loaded on the first factor and three items loaded on the second factor. All items on the first factor reflected the anticipation of relief from negative states (e.g. "even major problems in my life would not bother me if I drank/ used drugs now"). This factor was therefore labelled "expectancy of negative reinforcement”. Similarly, all items on the second factor reflected strong desires to use alcohol or drugs (e.g. "I would do almost anything to have a drink/ take some drugs right now"). This factor was therefore labelled "strong desires and intentions to drink or use drugs". The Pearson correlation between the factors was $r=0.69(p<0.01)$. Results of the factor analysis are summarised in Table 1. Overall these factors accounted for $81.76 \%$ of the overall variance ( $64.54 \%$ and $17.22 \%$ respectively). The scale mean was $2.80(S D=1.49)$, and the means for factors 1 and 2 were $3.01(S D=1.89)$ and $2.58(S D=1.49)$ respectively. Cronbach's alpha of 0.89 was found for the DAQ-6 in the present study, indicating a good level of internal consistency.

\section{[INSERT TABLE 1 HERE]}

\subsection{Convergent validity of the DAQ-6}

Both Spearman's and Pearson's correlations were conducted and results were examined to see whether results differed in terms of significance, direction and magnitude of relationships. Since results were highly consistent across both methods, the decision was made to report Pearson's correlations. Correlations were also conducted separately between the overall DAQ 
and each factor with a range of measures that are hypothesized to correlate positively and negatively with craving. As the pattern of results were highly consistent using the overall DAQ and individual factors, correlations using the overall DAQ-6 will be the focus of this study. Results have been reported in Table 2 .

\section{[INSERT TABLE 2 HERE]}

Consistent with the study's hypotheses, results indicated that the DAQ-6 correlated positively with measures of alcohol and drug severity (ASI) and psychological distress (DASS21), and negatively with psychological well-being (MHC-SF), alcohol and drug taking selfefficacy (DTCQ), recovery (RAS) and purpose in life (LET). Measures specific to alcohol and drug taking behaviour (ASI and DTCQ) demonstrated small correlations with craving, whilst those related to psychological distress (DASS-21 and MHC-SF) and other mental health measures (LET and RAS) demonstrated moderate correlations. None of these correlations were so high as to suggest that the DAQ-6 was assessing the same construct as these other measures. These findings suggest that the DAQ-6 has good convergent validity with other measures typically used in SUD contexts.

\section{Discussion}

The purpose of study 1 was to develop and examine the psychometric properties of a questionnaire suitable for assessing alcohol and drug craving in individuals in residential treatment settings. The DAQ-6 was based on the 36- and 14-item DAQ (Clark, 1994; Love et al., 1998), with two major modifications. First, items were chosen from the two dimensions that have most consistently emerged in prior studies. Second, items were worded such that they were relevant to both alcohol and drug users. 
As expected, factor analysis revealed that the 6-items were best described by a two-factor solution. These factors were labelled "expectancy of negative reinforcement" and "strong desires and intentions to drink or use drugs”. The DAQ-6 demonstrated a good level of internal consistency and items accounted for a similar, if not a greater amount of overall variance than reported in previous studies that included more items (Franken et al., 2002; James et al., 2002; Kramer et al., 2010; Love et al., 1998). This is consistent with our observations that while previous studies have reported up to four factors, only two factors have been consistently replicated. Correlations between the DAQ-6 and other measures typically used in SUD contexts were all significant and in the expected direction. Additionally, the pattern of correlations was similar across individual factors, suggesting that while the DAQ-6 captures more than one dimension of craving, these dimensions are related to other variables in very similar ways. Compared to previous studies which also conducted factor analysis on the DAQ, the only correlation that was smaller than expected in the present study was between craving and alcohol $(r=.15)$ and drug severity $(r=.22)$. One explanation is that both Kramer et al. (2010) and James et al. (2002) used questionnaires that are more focused on substance use symptom severity as opposed to severity based on the amount and frequency of alcohol and drug use. The correlations reported in the two studies were $r=0.72$ and $r=0.41-0.49$ respectively. In addition, they compared the DAQ with other self-report questionnaires administered at the same time increasing the likelihood that higher correlations are in part a function of shared method variance. In contrast, our study measured alcohol and drug use severity using the ASI which is an interview based measure. Further, the ASI asked respondents to report alcohol and drug use over the prior 30-days whereas cravings can potentially fluctuate daily or even hourly. Even with 
these method and timing differences we still found significant positive correlations between the DAQ-6 and drug and alcohol use measures.

The study upon which we primarily based our item selection only reported two factors. However, we recognise that some studies have reported a third "control” factor. Thus, for researchers and clinicians who are interested in the issue of perceived control, they may wish to include the following two control items: "I could easily limit how much alcohol/ drugs I would drink/ use if I drank/ used drugs now" and "I could easily limit how much alcohol/ drugs I drank/ used right now

\section{Study 2}

The results from study 1 demonstrated that the DAQ-6 adequately captures two factors of craving and explains a similar proportion of variance compared to the 36-item (Clark, 1996) and 14-item (Love et al., 1998) versions of the DAQ. Study 2 aimed to conduct confirmatory factor analysis on the DAQ-6 in an independent sample of participants, who were also undergoing residential treatment for substance use problems. Additionally, we assessed convergent/concurrent validity with an alternate measure of craving, as well as assessing the relationship of the DAQ-6 with other drug and alcohol measures.

6. Material and Methods

\subsection{Participants}

Data for the current study was collected from a study examining the effectiveness of a computer based, cognitive behavioral therapy treatment program delivered to participants attending residential treatment for alcohol and drug abuse operated by The Australian Salvation Army (Kelly et al., 2012). Participants who provided informed consent and who satisfied the 
diagnostic criteria for an alcohol or other substance dependence disorder were included in the study. Participants completed a baseline assessment prior to any intervention and were then randomly assigned to either a computer delivered substance abuse and depression intervention (treatment condition) or to the active control condition. Only data collected at baseline prior to the intervention was used for the purposes of Study 2. This sample comprised 116 participants. There were 94 males (81\%) and 20 females (17.2\%), with the average age being 38.19 $(S D=10.57)$ for both genders. The most frequent primary substance of use was alcohol for both genders (51.5\%) followed by amphetamines (11.3\%), cannabis (10.3\%), and heroin (9.3\%). The remaining participants were polydrug users. The average length of time participants indicated they had a problem with these substances was 15.78 years $(S D=11.42)$ for both genders.

\subsection{Measures}

In addition to the DAQ, DTCQ and DASS-21, the following measures were administered:

The Penn Alcohol Craving Scale (PACS) is a five item self-report questionnaire that explores the intensity, frequency and duration of craving throughout the past week (Flannery, Volpicelli, \& Pettinati, 1999). In the present study, the scale was modified to include alcohol and other drugs. For example, questions were modified to "During the past week how often have you thought about drinking or using, or about how good drinking alcohol or using drugs would make you feel?”. Cronbach’s alpha for the PACS has been reported as .92 (Flannery et al., 1999).

The Beck Depression Inventory Second Edition (BDI-II) is a 21 item self-report questionnaire that assesses depressive symptoms over the past two weeks (Beck, Steer, \& Brown, 1996). Participants were required to respond to a category by picking one statement of a group of statements that best describes the way they feel. For example, participants may respond 
to the category "sadness" by choosing a statement ranging from 0 ("I do not feel sad at all”) to 3 (“I am so sad or unhappy that I can’t stand it”). Internal consistency for the BDI-II is high, with coefficients ranging from .91 to .93 (Beck et al., 1996; Dozois, Dobson, \& Ahnberg, 1998).

\subsection{Statistical analyses}

Confirmatory factor analysis with maximum-likelihoods estimation method was conducted using the Analysis of Moment Structures (AMOS) version 17.0 software package. This CFA enabled us to test whether the two-factor model found in study 1 explained the structure of the empirical data collected in the second participant sample. Good model fit can be indicated by a non-significant chi-square statistic and several other fit statistics, including: the Goodness of Fit Index (GFI); the Tucker-Lewis Index (TLI); the Comparative Fit index (CFI); the Normed-Fit Index (NFI); and the Root Mean-Square Error of Approximation (RMSEA). When examining the GFI, TLI, CFI, NFI values less than .90 indicate lack of fit, values between .90 and .95 indicate reasonable fit and values between .95 and 1.00 indicate good fit. Root MeanSquare Error of Approximation values of .05 or lower to indicate good fit and values between .05 and .08 indicate reasonable fit (Byrne, 2001).

\section{Results}

The DAQ items were positively skewed so square-root and logarithmic transformations were employed to meet the assumption of normality. When using these transformed values in the

CFA the $\chi^{2}$ goodness of fit analyses was non-significant, $\chi^{2}(8, \mathrm{~N}=106)=15.17, \mathrm{p}=.056$, indicating that the amount of unexplained variance in the proposed two factor model was not significant. The additional CFA fit statistics also supported an adequate fit of the data to a two factor model $(\mathrm{CFI}=.986 ; \mathrm{NFI}=.972 ; \mathrm{TLI}=.974 ; \mathrm{RMSEA}=.092 ; \mathrm{GFI}=.957)$. Similar results were found when the model was run with the non-transformed data, $\chi^{2}(8, \mathrm{~N}=106)=14.46, \mathrm{p}$ 
$=.07(\mathrm{CFI}=.988 ; \mathrm{NFI}=.974 ; \mathrm{TLI}=.978 ; \mathrm{RMSEA}=.088 ; \mathrm{GFI}=.958)$. Figure 1 presents the results for the CFA with transformed data. Two latent constructs were present: desire and intention and negative reinforcement. The first three items of the DAQ predicted $5 \%$ of the variance in the urges construct while the remaining three items predicted $21 \%$ of the variance in the coping construct. The Pearson correlation between the two factors was $r=0.69(p<.01)$.

\section{[INSERT FIGURE 1 HERE]}

Table 3 provides correlations between the DAQ-6 and other measures. As expected, results indicated that the DAQ-6 correlated positively with measures of alcohol craving (PACS), depression (BDI-II) and psychological distress (DASS-21), and negatively with alcohol and drug refusal self-efficacy (DTCQ). Moderate to large correlations were found between the DAQ-6 and all measures. Importantly, the strongest correlation was found between the DAQ-6 and the PACS indicating good convergent validity with an alternate measure of craving. This was followed by strong correlations between craving and depression, psychological distress, and drug-taking refusal self-efficacy.

\section{[INSERT TABLE 3 HERE]}

\section{Discussion}

As predicted by Study 1, the CFA demonstrated that the DAQ-6 consists of two factors: “expectancy of negative reinforcement” and "strong desires and intentions to drink or use drugs". In addition, correlations between the DAQ-6 and other measures typically used in SUD contexts were all significant and in the expected direction, with minor differences between the DAQ-6 individual factors (subscales) and the total score. However, it should be noted that though the CFI, NFI, TLI and GFI values were all indicative of adequate model fit, the RMSEA value was 
slightly inflated. RMSEA values of .5 to .8 are generally considered to be indicative of adequate fit (Byrne, 2001). However, researchers should use multiple rather than single fit indices when determining model fit (Chen, Curran, Bollen, Kirby, \& Paxton, 2008; Byrne, 2001) and the RMSEA value tends to reject models when small sample sizes and few degrees of freedom are employed (Byrne, 2001; Hu \& Bentler, 1999). Thus, we do not believe the RMSEA value is indicative of poor model fit in our analyses.

The DAQ items compliment those provided by the PACS in several respects. The PACS is concerned primarily with quantifying the frequency, intensity, and duration of craving. However, the DAQ items focus more on qualitative experiences of craving, that is, the thoughts and feelings associated with craving. For example, in the two factor model, items are related to the reinforcing effects of using the substance and also the strong desire and intention to use.

We believe that the more qualitative perceptions tapped by items on the DAQ may be of additional utility in assessing cravings in residential settings. Although, it is unclear whether craving differs between residential and outpatient treatment centres, researchers have postulated that abstinent individuals in residential treatments may experience craving differently (Rohsenhow \& Monti, 1999). On the one hand, craving may be heightened because individuals are involuntarily prevented from substance use. On the other hand, craving may be infrequent and contained because individuals are not exposed to drug related stimuli that would normally provoke craving. Furthermore, there is evidence that drug availability influences the magnitude of craving, such that craving is enhanced when the individual believes they have immediate access to the desired drug (Carter \& Tiffany, 2001).

The frequency and intensity type items of the PACS may be more responsive to contexts where specific exposure to drugs and alcohol are more frequently present. The qualitative 
experience of cravings captured in the DAQ may be more related to perceptions of craving and appear less dependent on exposure to cues. At present this is speculative, but could be tested by comparing PACS and DAQ scores before and after exposure to situations that might cue cravings (e.g., walking past a bar).

In the current samples the two subscales of the DAQ-6 had similar correlations with other measures. The Negative Reinforcement and Desire and Intention subscales have potential clinical utility in treatment contexts. For example, the Negative Reinforcement subscale captures the extent to which respondents feel that drinking or drug use would provide relief from negative affect or life problems. Drinking to relieve distressing mood states has been repeatedly found (e.g., Holahan, Moos, Holahan Cronkite \& Randall, 2001; McDonald \& Meyer, 2011) and negative affect and craving cued relapse accounted for $68 \%$ of the reasons for relapse in the 12 months since alcohol treatment initiation on one study (Zywiak, Westerbert, Connors \& Maisto, 2003). All of the 85 clients who relapsed were at greatest risk within the first 2 months of treatment and the highest reasons for relapse in this window of time were negative affect followed by craving cued relapse (Zywiak et al., 2003). The negative reinforcement subscale has the potential to identify those individuals who may be more at risk of relapse due to craving focused on relief of negative emotional states. This provides the opportunities for treatment staff to not only identify risk of relapse but to adjust treatment content that might target alternative coping strategies to manage these negative emotional states or problems. The Desire and Intention subscale of the DAQ-6 reflects strong overwhelming wishes to drink or use drugs and that may be more akin to the craving-cued reason for relapse. It has been suggested that anticraving medications (e.g., naltrexone) may be particularly important to consider under such circumstances (Zywiak et al., 2003). To help determine the clinical utility of the two factors in 
the DAQ-6 it would be useful to administer the DAQ-6 regularly through residential treatment and then follow-up participants after discharge to monitor subsequent relapse. In addition, a measure of the reasons for drinking/drug use could be administered to clarify whether the DAQ6 subscales were able to also predict reasons for relapse.

\section{Limitations}

This study has two main limitations. Firstly, we recognize that the design of the study could have been strengthened if all items from longer versions of the DAQ were initially included before conducting exploratory factor analysis, and subsequent confirmatory factor analysis to obtain the resulting factor solution. Secondly, the measures we included to conduct concurrent validity were selected from a battery of routine assessments administered at Salvation Army alcohol and drug rehabilitation centres. These assessments were not necessarily specific measures that support the two factor structure of the DAQ; thus, further research is needed with more specific measures to further validate the two factors found in the 6-item version of the DAQ.

\section{Conclusion}

Overall, findings from the present study provide preliminary evidence that the DAQ-6 is a reliable and valid measure of craving in SUD populations. Given that the DAQ-6 includes substantially fewer items than both the 36- and 14-item versions, it provides a promising alternative in research and treatment contexts where questionnaires are often repeatedly administered and part of a larger battery of questionnaires. The brief nature of the measure combined with the capacity to tap into multiple components of craving provides a number of advantages over longer versions or brief measures that tap a single construct (e.g., PAC). A number of models of recovery from substance abuse theorise that successful management of 
cravings provides an important role in recovery (e.g. Niaura, 2000; Niaura et al., 1988; Witkiewitz \& Marlatt, 2007). The ability to take multiple measures of craving over time with minimal demand on participants offers the opportunity to monitor cravings during the treatment process to gauge progress. It is likely that this information will be useful to inform individual client care although further research is needed to assess the sensitivity of the cravings measure to changes over the course of treatment and their relationship with longer-term outcomes. 


\section{Acknowledgements}

The research was partially funded by a research consultancy to the second and fourth authors provided by The Salvation Army and a research grant provided by Rotary Health Australia. 


\section{References}

American Psychiatric Association. (2000). Diagnostic and statistical manual of mental disorders (4th ed., text rev.). Washington, DC: Author.

Anton, R. F., \& Drobes, D. J. (1998). Clinical measurement of craving in addiction. Psychiatric Annals, 28, 553-560.

Anton, R. F., Moak, D. H., \& Latham, P. (1995). The Obsessive-Compulsive Drinking Scale: A self-rated instrument of the quantification of thoughts about alcohol and drinking behavior. Alcoholism: Clinical and Experimental Research, 19, 92-99. doi: 10.1111/j.1530-0277.1995.tb01475.x

Baker, T. B., Piper, M. E., Fiore, M. C., McCarthy, D. E., \& Majeskie, M. R. (2004). Addiction motivation reformulated: An affective processing model of negative reinforcement. Psychological Review, 111, 33-51. doi:10.1037/0033-295X.111.1.33

Beck, A. T., Steer, R. A., \& Brown, G. K. (1996). Beck Depression Inventory manual (2nd ed.). San Antonio. TX: Psychological Corporation.

Byrne, B. M. (2001). Structural equation modelling with AMOS: basic concepts, applications, and programming. Mahwah, New Jersey: Lawrence Erlbaum Associates.

Carter, B. L. \& Tiffany, S. T. (1999). Meta-analysis of cue reactivity in addiction research. Addiction, 94, 327-340.

Clark, D. (1994). Craving for alcohol. Journal of Psychopharmacology, 9, 73. doi:10.1177/026988119500900114

Chen, F., Curran, P. J., Bollen, K. A., Kirby, J. \& Paxton, P. (2008). An empirical evaluation of the use of fixed cutoff points in RMSEA test statistic in structural equation models. Sociol Methods Res, 36, 462-494. doi:10.1177/0049124108314720. 
Corrigan, P. W., Salzer, M., Ralph, R. O., Sangster, Y., \& Keck, L. (2004). Examining the factor structure of the Recovery Assessment Scale. Schizophrenia Bulletin, 30, 10351041. doi:10.1093/oxfordjournals.schbul.a007118

Doherty, K., Kinnunen, T., Militello, F. S., \& Garvey, A. J. (1995). Urges to smoke during the first month of abstinence: Relationship to relapse and predictors. Psychomarmacology, 119, 171-178. doi:10.1007/BF02246158

Dozois, D. J. A., Dobson, K. S., \& Ahnberg, J. L. (1998). A psychometric evaluation of the Beck Depression Inventory-II. Psychological Assessment, 10, 83-89. doi: 10.1037/10403590.10.2.83

Drobes, D. J., \& Thomas, S. E. (1999). Assessing craving for alcohol. Alcohol Research \& Health, 23, 179-186.

Drummond, C. D., Litten, R. Z., Lowman, C. \& Hunt, W. A. (2000). Craving research: Future directions. Addiction, 95, 247-255. doi:10.1046/j.1360-0443.95.8s2.13.x

Flannery, B. A., Volpicelli, J. R., \& Pettinati, H. M. (1999). Psychometric properties of the Penn Alcohol Craving Scale. Alcoholism: Clinical and Experimental Research, 23, 1289-1295. doi: 10.1111/j.1530-0277.1999.tb04349.x

Franken, I. H. A., Hendriks, V. M., \& van den Brink, W. (2002). Initial validation of two opiate craving questionnaires: The Obsessive Compulsive Drug Use Scale and the Desires for Drug Questionnaire. Addictive Behaviors, 27, 675-685. doi:10.1016/S03064603(01)00201-5

Henry, J. D. \& Crawford, J. R. (2005). The short-form version of the Depression Anxiety Stress Scales (DASS-21): Construct validity and normative data in a large non-clinical sample. The British Journal of Clinical Psychology, 44, 227-239. doi:10.1348/01446650X29567 
Holahan, C. J., Moos, R. H., Holahan, C. K., Cronkite, R. C. \& Randall, P. K. (2001). Drinking to cope, emotional distress and alcohol use and abuse: A ten-year model. Journal of Studies on Alcohol,62, 190-198.

Hu, L. \& Bentler, P. M. (1999). Cutoff criteria for fit indexes in covariance structure analysis: conventional criteria versus new alternatives. Structural Equation Modeling, 6, 1-55. doi:10.1080/10705519909540118

James, D., Davies, G., \& Willner, P. (2004). The development and initial validation of a questionnaire to measure craving for amphetamine. Addiction, 99, 1181-1188. doi:10.1111/j.1360-0443.2004.00819.xKelly, P. J., Kay-Lambkin, F. J., Baker, A. L., Deane, F. P., Brooks, A. C., Mitchell, A., Marshall, S., Whittington, M., \& Dingle, G. A. (2012). Study protocol: A randomized controlled trial of a computer-based depression and substance abuse intervention for people attending residential substance abuse treatment. BMC Public Health, 12, 113. doi:10.1186/1471-2458-12-113

Kelly, P. J., Kay-Labkin, F. J., Baker, A. L., Deane, F. P., Brooks, A. C., Mitchell, A., Marshall, S., Whittington, M., \& Dingle, G. A. (2012). Study protocol: A randomized controlled trial of a computer-based depression and substance abuse intervention for people attending residential substance abuse treatment. BMC Public Health, 12, 113. doi:10.1186/1471-2458-12-113

Kozlowsk, L. T., \& Wilkinson, D. A. (1987). Use and misuse of the concept of craving by alcohol, tobacco and drug researchers. British Journal of Addiction, 82, 31-36. doi:10.1111/j.1360-0443.1987.tb01430.x 
Kramer, J. R., Chan, G., Hesselbrock, V. M., Kuperman, S., Bucholz, K. K., Edenberg, H. J., ... Porjesz, B. (2010). A principal components analysis of the abbreviated desires for alcohol questionnaire (DAQ). Journal of Studies on Alcohol and Drugs, 71, 150-155.

Lamers, S. M., Westerhof, G. J., Bohlmeijer, E. T., ten Klooster, P. M., \& Keves, C. L. (2011). Evaluating the psychometric properties of the Mental Health Continuum - Short Form (MHC-SF). Journal of Clinical Psychology, 67, 99-110. doi:10.1002/jclp.20741

Love, A., James, D., \& Willner, P. (1998). A comparison of two alcohol craving questionnaires. Addiction, 93, 1091-1102. doi:10.1046/j.1360-0443.1998.937109113.x

Lovibond, S. H., \& Lovibond, P. E. (1995). Manual for the Depression Anxiety Stress Scales (2nd ed.). Sydney: Psychology Foundation.

Marlatt, G. A. (1985). Relapse prevention: theoretical rationale and overview of model. In Marlatt, G. A., \& Gordon, G. (Eds.), Relapse prevention: A self-control strategy for the maintenance of behaviral change (pp. 3-70). New York: Guilford Press.

Marlatt, G. A. \& Gordon, J. R. (Eds.). (1985). Relapse prevention: Maintenance strategies in the treatment of addictive behaviors. New York: Guilford Press.

Marteau, T. M., \& Bekker, H. (1992). The development of a six-item short-form of the state scale of the Spielberger State-Trait Anxiety Inventory (STAI). British Journal of Clinical Psychology, 31, 301-306. doi:10.1111/j.2044-8260.1992.tb00997.x

McCormick, H. M., Horne, D. J. L., \& Sheather, S. (1988). Clinical applications of visual analogue scales: A critical review. Psychological Medicine, 18, 1007-1019. doi:10.1017/S0033291700009934 
McDonald, J. L., \& Meyer, T. D. (2011). Self-report reasons for alcohol use in bipolar disorders: Why drink despite the potential risks? Clinical Psychology and Psychotherapy, 18, 418425. doi:10.1002/cpp.782

McGregor, C., Srisurapanont, M., Jittiwutikarn, J., Laobhripatr, S., Wongtan, T., \& White, J. M. (2005). The nature, time course and severity of methamphetamine withdrawal. Addiction, 100, 1320-1329. doi:10.1111/j.1360-0443.2005.01160.x

McLellan, A. T., Kushner, H., Metzger, D., Peters, R., Smith, R., Grissom, G., \& Argeriou, M. (1992). The fifth edition of the addiction severity index. Journal of Substance Abuse Treatment, 9, 199-213. doi:10.1016/0740-5472(92)90062-S

McNaught, M., Caputi, P., Oades, L. G., \& Deane, F. P. (2007). Testing the validity of the Recovery Assessment Scale using an Australian sample. Australian and New Zealand Journal of Psychiatry, 41, 450-457. doi:10.1080/00048670701264792

Myers, M. G., McCarthy, D. M., MacPherson, L., \& Brown, S. A. (2003). Counstructing a short form of the Smoking Consequences Questionnaire with adolescents and young adults. Psychological Assessment, 15, 163-172. doi:10.1037/1040-3590.15.2.163

Niaura, R. (2000). Cognitive and social learning and related perspectives on drug craving. Addiction, 95, S155-S163. doi:10.1046/j.1360-0443.95.8s2.4.X

Niaura, R. S., Rohsenow, D. J., Binkoff, J. A., Monti, P. M., Pedraza, M. \& Abrams, D. B. (1988). Relevance of cue reactivity to understanding alcohol and smoking relapse. Journal of Abnormal Psychology, 97, 133-152. doi:10.1037/0021-843X.97.2.133

Rosenberg, H. (2009). Clinical and laboratory assessment of the subjective experience of drug craving. Clinical Psychology Review, 29, 519-534. doi:10.1016/j.cpr.2009.06.002 
Rohsehnow, D. J. \& Monti, P. M. (1999). Does urge to drink predict relapse after treatment? Alcohol Research \& Health, 23, 225-232. doi: 10.1046/j.1360-0443.1999.9433273.x

Sayette, M. A., Shiffman, S., Tiffany, S. T., Niaura, R. S., Martin, C. S. \& Shadel, W. G. (2000). The measurement of drug craving. Addiction, 95 (Suppl. 2), 189-210. doi:10.1046/j.13600443.95.8s2.8.x

Scheier, M. F., Wrosch, C., Baum, A., Cohen, S., Martire, L. M., Matthews, K. A., ... Zdaniuk, B. (2006). The Life Engagement Test: Assessing purpose in life. Journal of Behavioral Medicine, 29, 291-298. doi:10.1007/s10865-005-9044-1

Sklar, S. M., \& Turner, N. E. (1999). A brief measure for the assessment of coping self-efficacy among alcohol and other drug users. Addiction, 94, 723-729. doi:10.1046/j.13600443.1999.94572310.x

Sklar, S. M., Annis, H. M., \& Turner, N. E. (1999). Group comparisons of coping self-efficacy between alcohol and cocaine abusers seeking treatment. Psychology of Addictive Behaviors, 13, 123-133. doi:10.1037/0893-164X.13.2.123

Tiffany, S. T., \& Drobes, D. J. (1991). The development and initial validation of a questionnaire on smoking urges. British Journal of Addiction, 86, 1467-1476. doi:10.1111/j.13600443.1991.tb01732.x

Taylor, J., \& Deane, F. P. (2002). Development of a short form of the Test Anxiety Inventory (TAI). The Journal of General Psychology, 129, 127-136.

doi:10.1080/00221300209603133

Witkiewitz, K. \& Marlatt, G. A. (2007). High-risk situations: Relapse as a dynamic process. In K. Witkiewitz \& G. A. Marlatt (Eds.), Therapist's guide to evidence-based relapse prevention (pp. 19-33).Oxford, UK: Academic Press. 
World Health Organisation (1955). The craving for alcohol: a symposium by members of the WHO Expert Committees on Mental Health and on Alcohol. Quarterly Journal of Studies on Alcohol, 16, 33-66.

Zywiak, W. H., Westerberg, V. S., Connors, G. J., \& Maisto, S. A. (2003). Exploratory findings from the Reasons for Drinking Questionnaire. Journal of Substance Abuse Treatment, 25, 287-292. doi:10.1016/S0740-5472(03)00118-1 


\section{Table 1}

Factor loadings (PROMAX rotation, structure matrix) of the DAQ-6 in alcohol and drug dependent patients $(n=710)$.

$\begin{array}{ll}\text { Factor 1: } & \text { Factor 2: } \\ \text { Negative } & \text { Desire and } \\ \text { reinforcement } & \text { intention }\end{array}$

(5) Even major problems in my life would not bother me if

I drank/ used drugs now

0.97

0.48

(4) I would feel as if all the bad things in my life had

disappeared if I drank/ used drugs now

0.88

0.55

(6) I would feel less worried about my daily problems if I

drank/ used drugs now

0.86

0.53

(1) I want to drink/ use drugs so much I can taste it

0.52

0.93

(2) My desire to drink/ use drugs now seems

overwhelming

0.49

0.92

(3) I would do almost anything to have a drink/ take some

drugs right now

0.53

0.82 
Table 2

Pearson's Correlations of Desire for Alcohol Questionnaire (DAQ) with measures hypothesized to correlate with craving.

\begin{tabular}{cccc}
\hline \multirow{2}{*}{ Measure } & \multicolumn{3}{c}{ DAQ Subscales } \\
\cline { 2 - 4 } & $\begin{array}{c}\text { DAQ Factor 1 } \\
\text { Negative } \\
\text { reinforcement }\end{array}$ & $\begin{array}{c}\text { DAQ Factor 2 } \\
\text { Desire and intention }\end{array}$ & DAQ Total Score \\
\hline
\end{tabular}

\section{Alcohol Severity}

Index Alcohol

Composite $^{\mathrm{a}}$

$0.13^{*}$

$0.16^{* *}$

$0.15^{* *}$

$n$

340

340

340

Alcohol Severity Index Drug

Composite $^{b}$

$0.22 * *$

$0.20 * *$

$0.22 * *$

$n$

232

232

Drug Taking

Confidence

Questionnaire

$-0.28 * *$

$n$

379

$-0.29 * *$

379

$-0.31 * *$

379

Depression Anxiety

Stress Scale

$0.46 * *$

$0.41 * *$

$0.47 * *$

$n$

380

380

380

Mental Health

Continuum - Short

Form

$-0.36 * *$

309

$-0.36 * *$

$-0.38 * *$

$n$

309

309

Life Engagement Test

$-0.39 * *$

378

$-0.36 * *$

$-0.40 * *$

$n$

378

378

Recovery Assessment

Scale

$-0.40 * *$

382

$-0.39 * *$

$-0.40 * *$

$n$

382

382 
** Correlation is significant at the .001 level (two-tailed).

* Correlation is significant at the .05 level (two-tailed).

${ }^{a}$ Correlations were run for those who had alcohol as their primary drug.

${ }^{\mathrm{b}}$ Correlations were run for those who had drugs other than alcohol as their primary drug. 


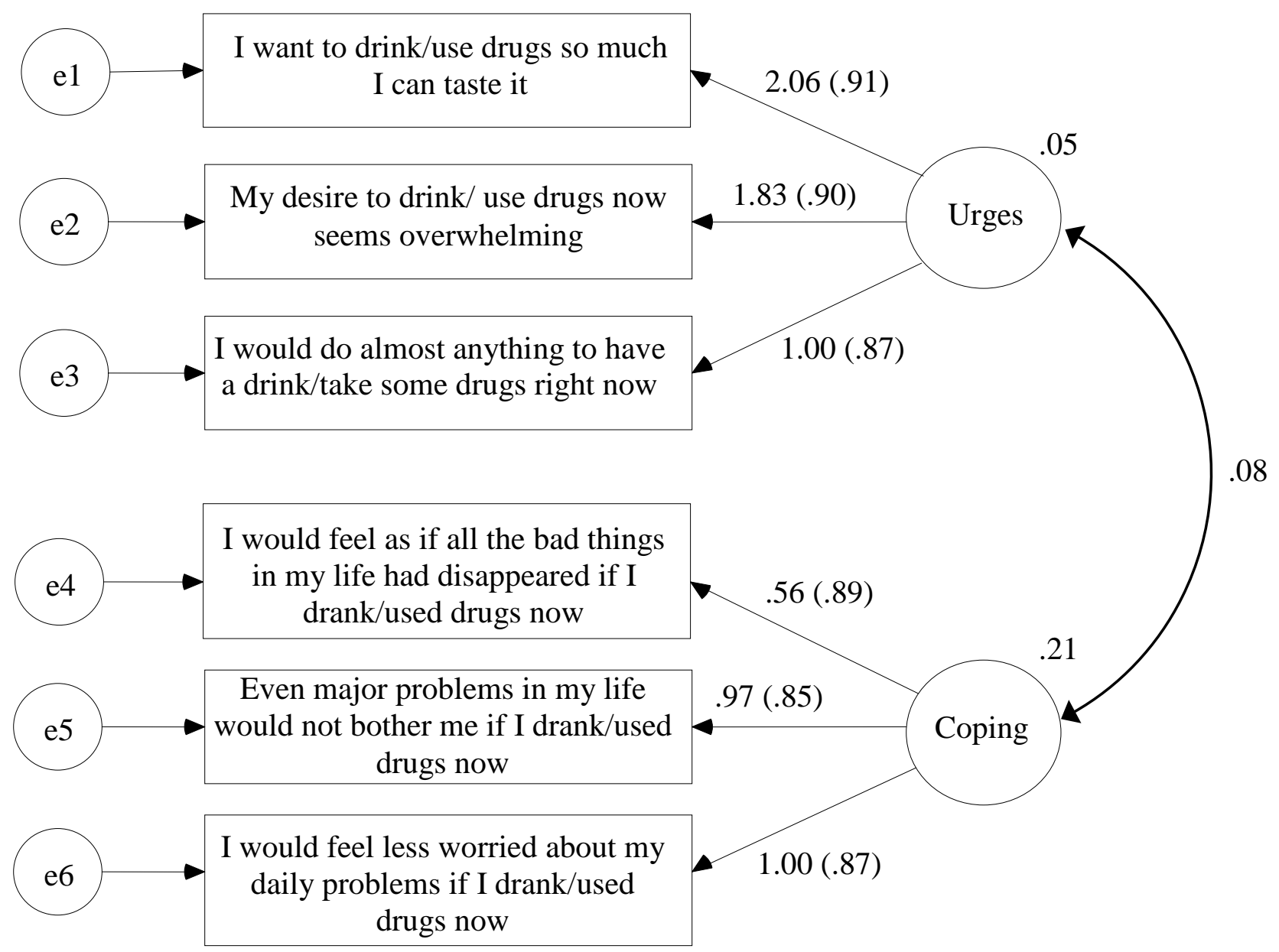

Figure 1. The two factor structure of the modified 6-item Desires for Alcohol Questionnaire (DAQ). Unstandardized coefficients are shown with standardized coefficients in parentheses. All values are significant at $p<.001$. 
Table 3

Pearson's Correlations of Desire for Alcohol Questionnaire (DAQ) with measures hypothesized to correlate with craving.

\begin{tabular}{lccc}
\hline & \multicolumn{3}{c}{ DAQ Subscales } \\
\cline { 2 - 4 } \multicolumn{1}{c}{ Measure } & $\begin{array}{c}\text { DAQ Factor 1 } \\
\text { Negative } \\
\text { reinforcement }\end{array}$ & $\begin{array}{c}\text { DAQ Factor 2 } \\
\text { Desire and } \\
\text { intention }\end{array}$ & $\begin{array}{c}\text { DAQ } \\
\text { Total } \\
\text { Score }\end{array}$ \\
\hline Penn Alcohol Craving Questionnaire & $0.72^{* *}$ & $0.60^{* *}$ & $0.72^{* *}$ \\
$n$ & 107 & 107 & 107 \\
Drug Taking Confidence Questionnaire & $-0.53^{* *}$ & $-0.56^{* *}$ & $-0.60^{* *}$ \\
$n$ & 108 & 108 & 108 \\
Depression Anxiety Stress Scale & $0.62^{* *}$ & $0.51^{* *}$ & $0.62^{* *}$ \\
$n$ & 107 & 107 & 107 \\
Beck Depression Inventory & & & $0.66^{* *}$ \\
$n$ & $0.64^{* *}$ & 108 & 108 \\
\hline$* *$ Correlation is significant at the .001 level (two-tailed). & &
\end{tabular}

